\title{
Effect of neutral leaks on pumping efficiency in 3D DEMO divertor configuration
}

\author{
Stylianos Varoutis ${ }^{\mathrm{a}}$, Yuri Igitkhanov ${ }^{\mathrm{a}}$, Christian Day $^{\mathrm{a}}$, Holger Strobel ${ }^{\mathrm{a}}$ and Ronald Wenninger ${ }^{\mathrm{b}, \mathrm{c}}$ \\ ${ }^{a}$ Karlsruhe Institute of Technology (KIT), Institute for Technical Physics, Karlsruhe, Germany \\ ${ }^{b}$ EUROfusion Programme Management Unit, Garching, Germany \\ ${ }^{c}$ Max-Planck-Institute for Plasma Physics (IPP), Garching, Germany
}

\begin{abstract}
In this paper we analyze the pumping efficiency of a generic 3D DEMO divertor configuration based on the size of inter-cassette gaps. Due to symmetry conditions we consider only one segment, which is composed by 3 divertor cassettes with the pumping port located at the bottom of the middle cassette and with four inter-cassette gaps. The width of intercassette gaps or the number of pumping ports is defined by the requirement to minimize the outflow of particles from the divertor to the plasma and hence, the highest possible pumping efficiency for the particle throughput, expected in DEMO, to be achieved. The DIVGAS code is used to perform a sensitivity analysis of the pumping efficiency, defined as a ratio of pumped particle flux to the particle throughput for different inter-cassette widths. The imposed neutral boundary conditions of pressure and temperature represent a moderate divertor pressure operating point. The analysis shows that for the reference case of $20 \mathrm{~mm}$ a reduction on the pumped flux of the order of $10 \%$ is observed when compared with the case of a completely sealed divertor. For smaller gaps the pumped flux reduction is found to be negligible, while for large values of the gap width the pumped flux reduction may reach the value of $20 \%$. Furthermore, almost $80 \%$ of the incoming particles are moving towards the $\mathrm{x}$-point, independent of the gap width. Therefore, the gap width does not influence the outflux towards the x-point, but only strongly affects the pumping efficiency. This analysis is meant to contribute to the ongoing DEMO divertor design efforts.
\end{abstract}

Keywords: DEMO reactor, divertor, pumping, fueling, neutral modelling

\section{Introduction}

One of the important aspects of DEMO divertor and the first wall design is the presence of gaps between the divertor cassettes, which cannot be sealed perfectly due to technical constraints. As a consequence, unintended particle fluxes will occur both in poloidal and toroidal direction and between the divertor cassettes. This issue has been already investigated for the case of Alcator CMod [1], in which the influence of the gaps in the poloidal and toroidal direction is significant and only if their existence is assumed, the corresponding numerical and experimental results may coincide. Furthermore, the same study has been performed for the case of ITER [2], where the simulations indicate that there is no major negative effect of the gaps on the divertor performance, although the parasitic flows caused by these gaps can be comparable to the pumping throughput. To the best of the authors' knowledge, there isn't any other study, which investigates the influence of the gaps in a systematic way and for several values of gap width. The only work, which describes in brief the influence of the gaps considering more than one gap width case, namely $10 \mathrm{~mm}$ and $20 \mathrm{~mm}$, is Ref. [3], which is focused on the ITER divertor. There, it has been found that for the burn phase a very strong back streaming of gas into the plasma, mainly caused by the flows in the gaps between the cassettes, takes place. Following the aforementioned studies, in the present work a three dimensional model of the DEMO divertor has been developed and simulated in order to investigate the influence of the gaps to the DEMO pumping efficiency, defined as a ratio of pumped particles to the total incoming particle throughput. The actual model of a DEMO divertor segment with the pumping port to be positioned at the bottom of the middle cassette is shown in Fig.1. The depicted divertor configuration is based on the 2015 EU DEMO baseline design [4], which consists of 54 divertor cassettes in total and has a major radius $R=9.1 \mathrm{~m}$. The presented configuration has been compared with the latest 2017 DEMO divertor design and no significant geometrical discrepancies, which may influence the outcome of this study, has been observed.

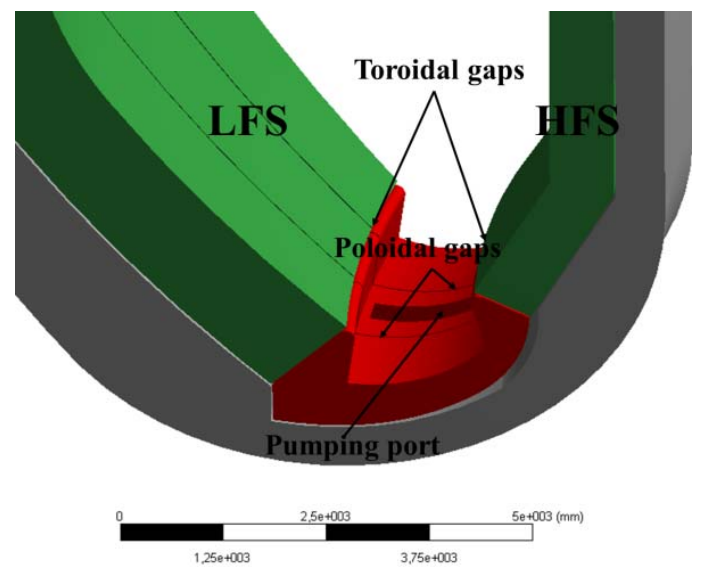

Fig. 1. Three dimensional DEMO 2015 divertor configuration including three divertor cassettes as well as the pumping port slot at the bottom, the first wall with low field side (LFS) and high field side (HFS) and the surrounding vacuum vessel. 
Due to toroidal symmetry we consider only one sector of $20^{\circ}$, which includes 3 divertor cassettes without the dome structure, two poloidal gaps between the cassettes (i.e inter-cassette gaps) and two toroidal gaps between the first wall and the divertor cassettes in both the low field and high field sides (LFS and HFS). Furthermore, a pumping port is considered and is located in the middle cassette occupying the whole bottom part of the structure. All the gaps are interconnected and lead to the pump entrance, which is located below the end of the pumping port. In the present work, the width of all aforementioned gaps consists of the main input parameter of the analysis and is defined by the requirement to minimize the outflow of particles from the divertor into the plasma and hence, the highest possible pumping efficiency to be achieved in DEMO.

The primary goal of this paper is to model the gas flow in the volume, which extends between the divertor cassettes, the vacuum vessel and the first wall and to explore the effect of the neutral flux through the gaps onto the DEMO divertor pumping efficiency and the possible need of additional sealing. For this purpose, the DIVGAS code [5], which describes the neutral particle transport for arbitrary collisionality regime, has been implemented. The code is based on the Direct Simulation Monte Carlo (DSMC) method [6] and estimates the distribution of macroscopic parameters of neutrals in the whole flow domain as well as the flow pattern, the pumped particle flux and the particle flux through the inter-cassette gaps and through the private flux region towards the plasma.

The analyses of the influence of the inter-cassette gaps on the overall pumping efficiency are meant to contribute to the ongoing DEMO divertor design efforts. The schematic model taking into account the gas leaks between the divertor components is described in section 2 , the $3 \mathrm{D}$ modelling results upon application to DEMO are shown in section 3 , and conclusions are drawn in section 4.

\section{Numerical model}

\subsection{The 3D divertor geometry}

In the present work five different geometrical configurations were considered (see Fig. 1), namely a configuration without gaps with gap width $d=0 \mathrm{~mm}$ (i.e. completely sealed divertor) and four configurations, in which the gap width $d$ in the poloidal and toroidal direction is finite and takes the following values: $10 \mathrm{~mm}$, $20 \mathrm{~mm}, 30 \mathrm{~mm}$ and $40 \mathrm{~mm}$.

In Fig. 2, the applied 3D DEMO divertor configuration, which corresponds to a $20^{\circ}$ sector and includes the toroidal and poloidal gaps, is shown. For representation purposes, the plane $\mathrm{KK}^{\prime}$ is additionally introduced. It is noted that in all simulations the pumping port is considered in the middle of the second cassette and occupies the whole bottom divertor surface. The choice of the pumping port position, located in the middle of the divertor cassette and not in the LFS or HFS, is based on the outcome of our previous recent investigation [7], which indicates that this position is the optimum one in terms of the divertor pumping efficiency.

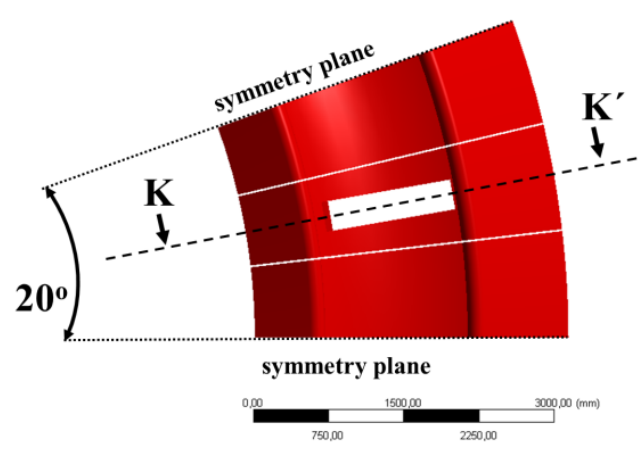

Fig. 2. A $20^{\circ}$ DEMO divertor sector is presented. The plane $\mathrm{KK}^{\prime}$ as well as the symmetry planes are shown.

In Fig. 3, a $2 \mathrm{D}$ cut along the $\mathrm{KK}^{\prime}$ plane of the $3 \mathrm{D}$ model is presented. In this specific design, the particles, which enter the pumping port and reach its outlet, have the possibility to flow into the toroidal or poloidal direction in the volume between the divertor cassette and the vacuum vessel. The adsorbing surface $D$ named as pumping slot in this case is located further below the pumping port outlet and a given capture coefficient $\xi$ is appointed. The transparent surfaces $A$ and $B$ in the HFS and LFS correspond to the virtual windows, through which neutrals penetrate the private flux region and on which the incoming boundary conditions are imposed. The corresponding area for each surface is $1.6 \mathrm{~m}^{2}$ and $2.8 \mathrm{~m}^{2}$ respectively. The surface $C$ represents the boundary through which the neutrals will be immediately ionized and removed from the flow domain and it is extended to the toroidal direction. The area of surface $C$ is $1.7 \mathrm{~m}^{2}$. The surfaces $E$ and $F$ correspond to the outlet of the toroidal gaps.

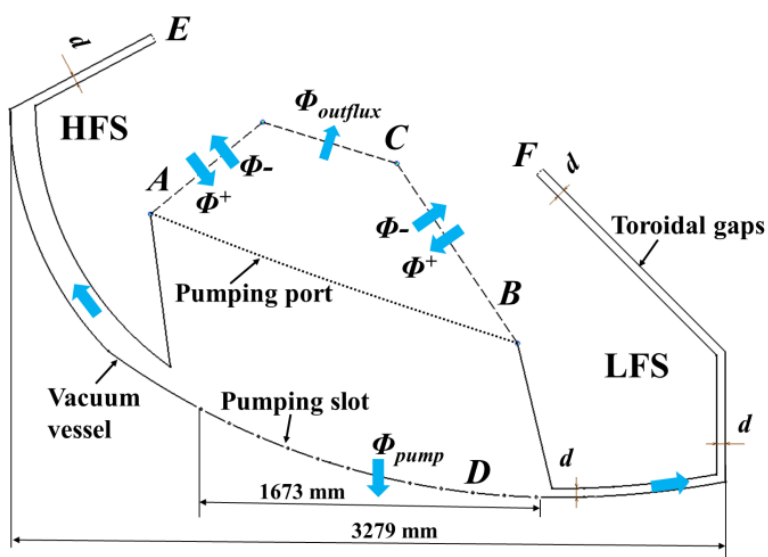

Fig. 3. A $2 \mathrm{D}$ cut along the $\mathrm{KK}^{\prime}$ plane of the $3 \mathrm{D}$ DEMO divertor numerical model.

In Fig. 4a, a representation of the cut along the KK' plane from another perspective, is shown. In this cut the considered toroidal as well as the poloidal gaps between the divertor cassettes are depicted. A bottom view angle of the model is shown in Fig 4b, at which the pumping slot is highlighted. The total area of the pumping slot is equal to $2.1 \mathrm{~m}^{2}$. 

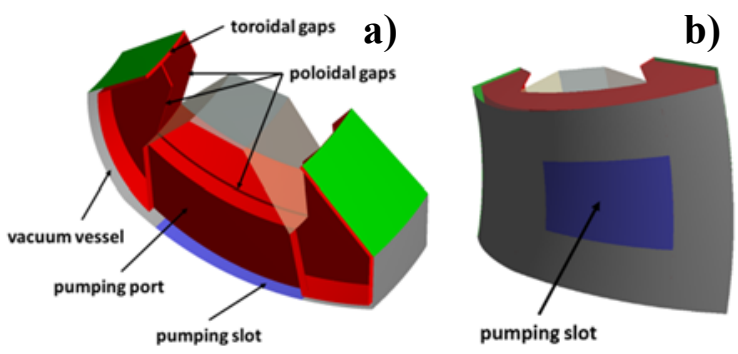

Fig. 4. a) A cut along the plane $\mathrm{KK}^{\prime}$ (shown in Fig. 2), in which the toroidal and poloidal gaps, the vacuum vessel, the pumping port and the pumping slot are shown. b) A bottom view angle of the model, in which the location of the pumping slot is shown.

\subsection{Numerical approach and boundary conditions}

Since DSMC has been proven to be a reliable numerical tool to describe the behavior of rarefied gases in the divertor area, detailed description of this method here is omitted and the reader may refer to the following publications [7-9] for further details. In the present work only the essential aspects of the implementation are mentioned. Briefly, the simulated number of particles for all calculations ranges between $10^{7}$ to $2 \times 10^{7}$, while the time step is equal to $\Delta t=1 \mu \mathrm{s}$. For all cases, an unstructured tetrahedral mesh has been implemented. The total number of the computational cells is of the order of $2 \times 10^{6}$. It is noted that all the chosen values related to the number of simulated particles, the time step $\Delta t$ and the size of the computational grid, have been chosen so as an invariance in the results of less than $5 \%$ to be achieved. Each simulation needs several weeks for reaching steady state conditions by using an HPC and in particular 200 CPUs for each run. For all simulated cases the particle balance of the total incoming and outgoing particles in the flow domain is satisfied.

In the present model, the neutral deuterium flux entering the private flux region is defined by specifying the pressure and the temperature at the entrance to the private flux region (PFR). In particular, it is assumed that all the plasma deuterium ions are neutralized and recombined on the vertical divertor plates and as $\mathrm{D}_{2}$ molecules penetrate the PFR (He or T molecules can be treated in similar fashion in the model) with reference pressure $P_{0}$ and temperature $T_{0}$ equal to $1 \mathrm{~Pa}$ and $4023 \mathrm{~K}$ (or $0.35 \mathrm{eV}$ ) respectively. These macroscopic quantities define the incoming neutral flux $\Phi^{+}$, as shown in Fig. 3. The outgoing neutral fluxes $\Phi^{-}, \Phi_{\text {outflux }}$ and $\Phi_{\text {pump would }}$ be an outcome of each simulation. The applied boundary conditions of pressure and temperature for molecular deuterium reflect a moderate divertor pressure scenario (i.e. similar to an ITER semi-attached low density regime), for which the needed computational time still remains reasonable. It is noted that in the present work the ionization, dissociation and recombination processes on the walls or in the gas phase are not taken into account. Therefore, when a deuterium molecule hits a stationary wall, it remains as molecule and a purely diffuse reflection takes place, where the incident molecule is reemitted with Maxwellian distribution based on the wall temperature, equal to $T_{\text {wall }}=420 \mathrm{~K}$. In the case of a particle intersecting with the pumping slot (i.e. dashed dotted line $D$ in Fig. 3), it can be completely removed from the computational domain according to a certain probability, which is given by the capture coefficient $\xi$. Consequently, $\xi$ takes values between $0 \leq \xi$ $\leq 1$. It is noted that $\xi$ represents the imposed condition of fixed pumped particle flux and is related with the effective pumping speed of the pumping slot. In the present work the capture coefficient takes the following values, namely $\xi=0.1,0.3,0.6$ and 1.0 (while in reality low $\xi$ values between 0.1 and 0.3 should be expected). If the particle is not finally absorbed from the pumping surface, then the particle undergoes a diffuse reflection, assuming that the temperature at the entrance to the pump is equal to $420 \mathrm{~K}$. Additionally, when a particle moves through the toroidal and poloidal gaps (shown in Fig. 4a) as well as the surface $C$ (shown in Fig. 3) then, an expansion into vacuum is considered resulting in the complete particle removal. The latter assumption can be justified, since an interaction with the plasma takes place and results in the immediate neutral particle ionization. Finally, when a particle intersects with one of the symmetry planes, as shown in Fig. 3, then the particle undergoes a specular reflection and preserves its kinetic energy.

\section{Results and discussion}

In Fig. 6, the normalized fraction of fuel particles pumped out as a function of gap width is shown for four values of the capture coefficient $\xi$. It is seen that, as the gap width increases, the pumped out fraction decreases. In particular, for the reference case of a $20 \mathrm{~mm}$ gap, the normalized pumped flux reduction is maximum $10 \%$ and depends on $\xi$. For the case of $10 \mathrm{~mm}$ width, it is observed that the normalized pumped flux reduction weakly depends on $\xi$. In addition, for gap width less than $10 \mathrm{~mm}$, the normalized pumped flux is not affected by the gap size for any value of $\xi$. For larger values of gap width, the normalized pumped flux reduction is more significant and a maximum value of $20 \%$ was found for gap width of $40 \mathrm{~mm}$ and $\xi=0.1$. It is noted that the estimated ratio does not take into account the particle losses towards the $\mathrm{x}$-point.

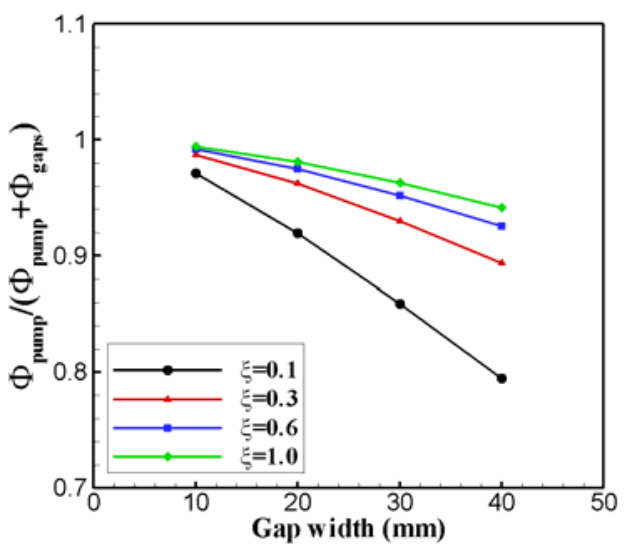

Fig. 6. The normalized pumped flux of fuel particles as a function of the gap width and various values of $\xi$.

In Fig. 7, the ratio of the total particle flux going through the gaps and back to SOL to the pumped out flux in the case without gaps as a function of the gap 
width is presented. This ratio represents the influence of the gaps in the pumping efficiency and in general the higher this ratio, the smaller the divertor pumping efficiency. More specific, it is observed that in the reference case of $20 \mathrm{~mm}$, the ratio varies between $2 \%$ and $15 \%$, which shows that in the worst case $15 \%$ of the particle flux flows through the gaps and penetrates the SOL. The rest of the particles either will be pumped out or move towards the x-point. In the case of $40 \mathrm{~mm}$ gap and for $\xi=0.1$, the ratio is equal to $50 \%$, which means that half of the total incoming particles will flow back to the SOL and will not be finally pumped. In reality, it is expected that there is going to be an influence of the escaped particles to the incoming boundary conditions, but such an effect will be considered only in a future investigation.

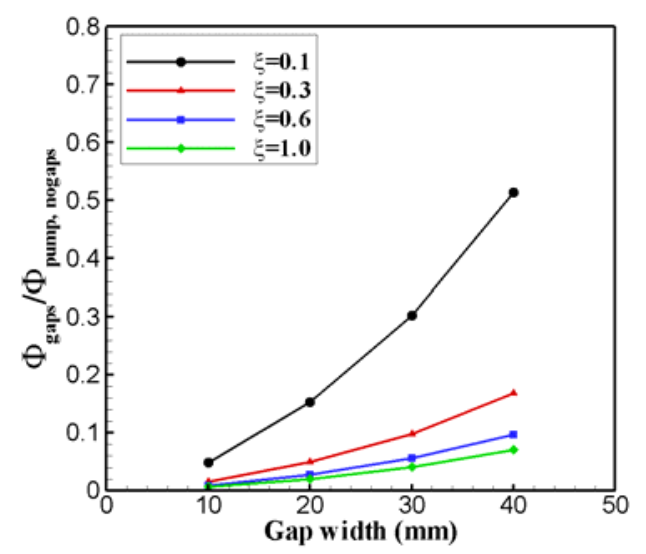

Fig. 7. Ratio of particle flux through the gaps and the pumped flux in the case without the gaps as a function of gap width and various values of $\xi$.

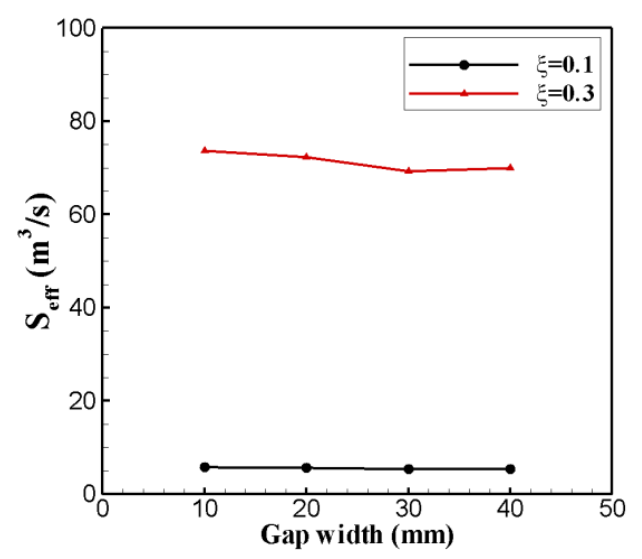

Fig. 8. Effective pumping speed $S_{\text {eff }}$ as a function of gap width and two values of $\xi$.

In Fig. 8, the effective pumping speed $S_{\text {eff }}$ of the studied configuration defined as

$$
S_{\text {eff }}=\Phi_{\text {pump }} / P_{\text {aver }}
$$

is presented in terms of the gap width and two values of $\xi$. In Eq. (1) $\Phi_{\text {pump }}$ is the pumped flux through the pumping slot (see Fig. 4b) in $\mathrm{Pa} \mathrm{m}^{3} / \mathrm{s}$ and $P_{\text {aver }}$ is the average pressure in $\mathrm{Pa}$, estimated on the whole pumping slot area according to

$P_{\text {aver }}=\iint P(x, y) d A$.
It is seen that there is a weak dependence of $S_{\text {eff }}$ on the gap width for any $\xi$. On the contrary, a strong dependence of $S_{\text {eff }}$ on $\xi$ is observed for any gap width. For the reference case of $20 \mathrm{~mm}$ and $\xi=0.1$ the effective pumping speed of the complete divertor ring (i.e 54 cassettes with 18 pumping slots in total) results to 101 $\mathrm{m}^{3} / \mathrm{s}$.

In Fig. 9, the normalized outflux of particles, which escape the flow domain and move towards the x-point, as a function of gap width is depicted. The normalization of outflux is based on the total net particle flux, which enters the PFR. It is clearly seen that for the given configuration without the dome, the outflux is almost independent of the gap width and the $\xi$. Here it should be noted that the imposed boundary conditions are kept fixed, whereas in principle the particles, which escape to the PFR, affect the incoming boundary conditions by considering their reentry to the PFR. A more realistic representation would be to assume variable incoming boundary conditions of molecular deuterium, which will lead to the increase of the outflux and the corresponding pumped flux. Since the particle flux through PFR boundaries back to plasma prevails all other particle losses in the divertor, the actual dependence of pumping efficiency on the gap width is not so critical. For the reference case of $20 \mathrm{~mm}$, the outflux is $\sim 82 \%$ of the total net incoming to PFR particle flux. This conclusion indicates that there is a necessity towards a study, which takes into account the dome structure in order a more precise definition of the actual role of gaps on the pumping efficiency to be assessed.

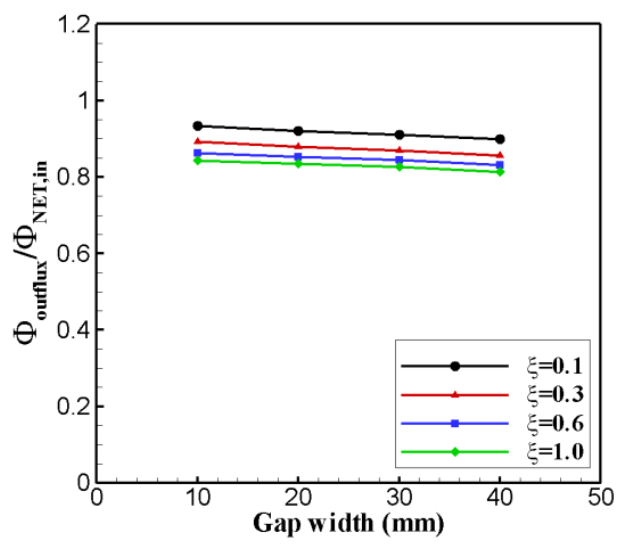

Fig. 9. Normalized particle outflux as a function of gap width for various values of $\xi$.

In Fig. 10, a representative pressure contour and the corresponding streamlines for the case of a gap width of $40 \mathrm{~mm}$ and a capture coefficient of $\xi=1$ are presented. It is seen that the particle move is fully justified by the local pressure drop. The particle flow creates a very complex pattern, which is extended around the divertor cassettes and through the divertor gaps. The present flow case is an indicative example, while the same qualitative behavior was found in the other simulations as well. 


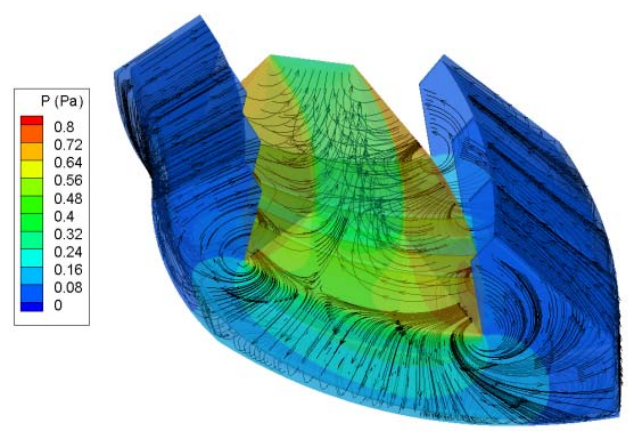

Fig. 10. Pressure contour and streamlines in the whole flow field, for the case of gap width $40 \mathrm{~mm}$ and capture coefficient equal to $\xi=1$.

\section{Conclusions}

The results of DIVGAS modelling of the three dimensional 2015 DEMO divertor design is considered in the present work. The chosen boundary conditions for molecular deuterium reflect a moderate divertor pressure scenario (i.e. similar to an ITER semi-attached low density regime), for which the needed computational time remains reasonable. The effect of gas leaks between the divertor cassettes is assessed and a sensitivity analysis on the pumping efficiency by varying the intercassette gap width is being conducted.

The analysis shows that for the reference case of 20 $\mathrm{mm}$ a reduction on the pumped flux up to $10 \%$ is obtained for small values of capture coefficient $\xi$. For smaller values of the gap width the pumped flux reduction was found to be negligible and almost independent of the gap width and $\xi$, while for larger values of the gap width the pumped flux can be reduced up to $20 \%$ for a $40 \mathrm{~mm}$ gap and small $\xi$.

In the present configuration almost $80 \%$ of the incoming to PFR particles are moving towards the $\mathrm{x}$ point, independent of the gap width. Therefore, the gap width does not influence the outflux towards the x-point, but only affects the pumping efficiency. Previous work conducted for the ITER divertor with dome revealed similar conclusions [2]. Hence, as future work, the same parametric investigation will be performed, by taking into account the dome structure. Consequently, the influence of the outflux will be eliminated and the effect of gaps on the pumping efficiency will be revealed in a more pronounced way. The analyses of the influence of the inter-cassette gaps on pumping efficiency are meant to contribute to the ongoing DEMO divertor design efforts.

\section{Acknowledgments}

This work has been carried out within the framework of the EUROfusion Consortium and has received funding from the Euratom research and training programme 2014-2018 under grant agreement No 633053. The views and opinions expressed herein do not necessarily reflect those of the European Commission.

Part of this work was performed by using computational resources provided by the MARCONIFUSION HPC. The rest of this work was performed on the computational resource bwUniCluster funded by the
Ministry of Science, Research and Arts and the Universities of the State of Baden-Württemberg, Germany, within the framework program bwHPC.

\section{References}

[1] D.P. Stotler, B. LaBombard, "Three-dimensional simulation of gas conductance measurement experiments on Alcator C-Mod", J. Nucl. Mater. vols. 337-399, pp. 510-514, 2005.

[2] A.S. Kukushkin, H.D. Pacher, V. Kotov, D. Reiter, D. Coster and G.W. Pacher, "Effect of conditions for gas recirculation on divertor operation in ITER", Nucl. Fusion, vol. 47(7), pp. 698-705, 2007.

[3] V. Hauer, Chr. Day, "ITER divertor gas flow modelling", FED, vols. 98-99, pp. 1775-1778, 2015.

[4] R. Wenninger et al., "The physics and technology basis entering European system code studies for DEMO”, Nucl. Fus., vol. 57, pp. 016011, 2017

[5] S. Varoutis et al., "Simulation of neutral gas flow in the JET sub-divertor", FED, vol. 121, pp. 13-21, 2017.

[6] G. A. Bird, Molecular Gas Dynamics and the Direct Simulation of Gas Flows, Oxford University Press, Oxford, UK, 1994.

[7] S. Varoutis et al., "Optimization of pumping efficiency and divertor operation in DEMO", Nucl. Materials and Energy, doi:10.1016/j.nme.2017.04.001, 2017.

[8] F. Bonelli et al., "Self-consistent coupling of DSMC method and SOLPS code for modeling tokamak particle exhaust", Nucl. Fusion, vol. 57(6), pp. 066037, 2017.

[9] Chr. Day, S. Varoutis and Yu. Igitkhanov, "Effect of the dome on the collisional neutral gas flow in the DEMO divertor", IEEE Trans. Plasma Sc., vol. 44(9), pp. 1636-1641, 2016. 